\title{
Análise Comparativa da Tecnologia Fotovoltaica Aplicada em microgeração fixa e sistema tracker com o mínimo de engrenagens.
}

\author{
Comparative Analysis of Applied Photovoltaic Technology in fixed micro-generation and \\ tracker system with the minimum of gears.
}

\section{Carlos Alexandre de Sá Ribeiro ${ }^{1,2}$ (Dorcid.org/0000-0001-9868-7194}

\section{José Bione de Melo Filho',2 (1D orcid.org/0000-0002-9283-3362}

\footnotetext{
${ }^{1}$ Escola Politécnica de Pernambuco, Universidade de Pernambuco, Recife, Brasil,

2 Pós-graduação em Energias Solar e Eólica, Escola Politécnica de Pernambuco, Pernambuco, Brasil,

E-mail do autor principal: Carlos Ribeiro carlossr80@gmail.com
}

\section{Resumo}

O presente artigo, tem como objetivo mensurar o ganho de eficiência em um sistema de painel solar seguidor com um sistema fixo tradicional. Poderemos ver o aumento da eficiência de captação solar em painéis fotovoltaicos com um sistema tracker, usando o mínimo de engrenagens e a partir de software aberto, gratuito e de boa eficiência. Para realização desta análise de eficiência usaremos o software PVsyst. A plataforma usada para automação do tracker será feita através do Arduino. Aplicando as diretrizes de movimento sugeridas pelo PVsyst poderemos observar um ganho de até $30 \%$ de geração de energia, tomando como parâmetros um projeto sendo aplicado em Gravatá -PE. Com a nossa proposta de usar o mínimo de engrenagens possível no sistema tracker, reduziremos consideravelmente manutenções e defeitos deste sistema.

Palavras-Chave: Tracker solar, Eficiência energética, Painel fotovoltaico;

\begin{abstract}
The aim of this article is to measure the efficiency gain in a follower solar panel system with a traditional fixed system. We can see the increased efficiency of solar pickup in photovoltaic panels with a tracker system, using the minimum of gears and from open, free and good efficiency software. To perform this efficiency analysis we will use the PVsyst software. The platform used for automation of the tracker will be done through the Arduino. Applying the movement guidelines suggested by PVsyst we can observe a gain of up to $30 \%$ of power generation, taking as parameters a project being applied in Gravatá -PE. With our proposal to use as few gears as possible in the tracker system, we will considerably reduce maintenance and defects of this system.
\end{abstract}

Key-words: Solar Tracker, energy efficiency, photovoltaic panel; 


\section{Introdução}

Devido ao novo estilo de vida adotado pela sociedade moderna, cada vez mais industrializada e ao grande crescimento da população, a demanda de energia elétrica é cada vez maior[1]. Sendo assim, o consumo de energia vem apresentando um crescimento acelerado. Simultaneamente, a sociedade se movimenta no sentido de se conscientizar das alterações ocasionadas na natureza. Os combustíveis fósseis foram sempre os principais componentes da matriz energética mundial. O aumento da demanda mundial por energia e os impactos causados por essas fontes fizeram com que os países começassem a buscar fontes alternativas de geração de energia elétrica para substituir as fontes fósseis. Ao longo do tempo, a energia solar tem sido cada vez mais visada pelos pesquisadores por ser uma fonte de energia inesgotável e limpa.

Dentre as várias tecnologias existentes que podem ser usadas para aumentar o rendimento dos módulos fotovoltaicos, encontram-se os seguidores solares. $O$ interesse por essa tecnologia deve-se ao fato de ser uma tecnologia relativamente simples e que permite, na maioria dos casos, aumentos significativos na produção, em troca de um investimento relativamente baixo, quando comparado com o custo dos painéis fotovoltaicos.

A geração de energia fotovoltaica no Brasil ainda é muito pouco explorada, comparada com as demais fontes geradoras, mesmo contando com um grande potencial pela sua localização geográfica no planeta. A geração fotovoltaica é uma tecnologia ainda cara dependendo da aplicação; portanto, mesmo em sistemas relativamente pequenos, na maioria das vezes há vantagem econômica na utilização de rastreadores. A vantagem se torna ainda maior se 0 rastreador for associado com 0 uso de concentradores solares fixos (BIONE et al.,2004). No Brasil, já são realidade os sistemas de geração conectados à rede de distribuição elétrica, graças à Resolução Normativa da ANEEL n.o $482 / 2012$, que permite ao consumidor ser também o produtor. Nesse sistema, o consumidor pode gerar energia elétrica em sua residência e transmiti-la para distribuidora, recebendo, por isso, um abatimento em sua conta de energia consumida. Por meio de um medidor bidirecional, sabe-se quanto de energia foi injetada na rede e o quanto foi consumida [2].
Por ser uma energia limpa e, praticamente, inesgotável, que pode ser aproveitada através de várias tecnologias, desde a solar térmica até a solar fotovoltaica. Acredita-se que tal tecnologia venha a tornar-se uma das fontes energéticas com maior crescimento futuro. O seguidor solar controlado por um micro-controlador arduino, através de um motor de passo usando um sistema de correias que dispensa o uso de roldanas e engrenagens, serão alvo de estudos neste artigo.

O Arduino foi criado em 2005 por um grupo de 5 pesquisadores : Massimo Banzi, David Cuartielles, Tom Igoe, Gianluca Martino e David Mellis. O objetivo era elaborar um dispositivo que fosse ao mesmo tempo barato, funcional e fácil de programar, sendo dessa forma acessível a estudantes e projetistas amadores. Além disso, foi adotado o conceito de hardware livre, o que significa que qualquer um pode montar, modificar, melhorar e personalizar o Arduino, partindo do mesmo hardware básico [3].

Os Motores de Passo são dispositivos eletromecânicos que convertem pulsos elétricos em movimentos mecânicos que geram variações angulares discretas. $O$ rotor ou eixo de um motor de passo é rotacionado em pequenos incrementos angulares, denominados "passos", quando pulsos elétricos são aplicados em uma determinada seqüência nos terminais deste. A rotação de tais motores é diretamente relacionada aos impulsos elétricos que são recebidos, bem como a sequência a qual tais pulsos são aplicados reflete diretamente na direção a qual o motor gira. A velocidade que o rotor gira é dada pela frequência de pulsos recebidos e o tamanho do ângulo rotacionado é diretamente relacionado com o número de pulsos aplicados [4].

\section{MATERIAIS E MÉTODOS}

Para a análise comparativa da tecnologia fotovoltaica de sistema fixo e com tracker de uma central geradora de $10 \mathrm{kWp}$, foi escolhida a cidade de Gravatá-PE $\left(-8.1841^{\circ} \mathrm{S}\right.$ e $\left.-35.5847^{\circ} \mathrm{W}\right)$ por possuir uma posição estratégica no agreste e sertão do estado e os dados de valores médios de irradiação solar utilizados na simulação foram do Meteonorm 7.2. Onde por sua vez, todos os 
parâmetros usados para o projeto foram analisados na plataforma do PvSystem em sua versão 6.8 .

As simulações foram feitas em duas etapas: uma com o sistema com string fixo e outra com a utilização do tracker. Mantivemos exatamente a mesma configuração de material para as duas simulações para podermos fazer uma comparação mais fiel em termos de ganhos com o sistema tracker.

Detalharemos na tabela 1 as configurações feitas para os dois processos:

\begin{tabular}{|c|c|c|c|c|c|}
\hline \multicolumn{6}{|c|}{ Sistema SEM Tracker } \\
\hline $\begin{array}{l}\text { Modelo } \\
\text { das } \\
\text { Placas }\end{array}$ & $\begin{array}{l}\text { Modelo } \\
\text { do } \\
\text { Inversor }\end{array}$ & $\begin{array}{l}\text { Inclinação } \\
\text { Das strings }\end{array}$ & $\begin{array}{l}N^{\circ} \text { de } \\
\text { strings }\end{array}$ & $\begin{array}{l}\mathrm{N}^{\circ} \text { de } \\
\text { Placas }\end{array}$ & $\begin{array}{c}\text { Potência } \\
\text { Gerada } \\
\text { (Com } \\
\text { perdas) }\end{array}$ \\
\hline $\begin{array}{c}\text { CS3K } \\
27 \mathrm{P} \\
1500 \mathrm{~V}\end{array}$ & $\begin{array}{l}\text { StecaGrid } \\
10000 \\
+3 \mathrm{ph}\end{array}$ & $10^{\circ}$ (Norte) & 2 & 36 & $\begin{array}{c}\text { 12,03MW } \\
\text { /Ano }\end{array}$ \\
\hline \multicolumn{6}{|c|}{ Sistema COM Tracker } \\
\hline $\begin{array}{l}\text { Modelo } \\
\text { das } \\
\text { Placas }\end{array}$ & $\begin{array}{l}\text { Modelo } \\
\text { do } \\
\text { Inversor }\end{array}$ & $\begin{array}{l}\text { Inclinação } \\
\text { Das strings }\end{array}$ & $\begin{array}{l}N^{\circ} \text { de } \\
\text { strings }\end{array}$ & $\begin{array}{l}\mathrm{N}^{\circ} \text { de } \\
\text { Placas }\end{array}$ & $\begin{array}{c}\text { Potência } \\
\text { Gerada } \\
\text { (Com } \\
\text { perdas) }\end{array}$ \\
\hline $\begin{array}{c}\text { CS3K } \\
27 \mathrm{P} \\
1500 \mathrm{~V}\end{array}$ & $\begin{array}{l}\text { StecaGrid } \\
10000 \\
+3 \mathrm{ph}\end{array}$ & $\begin{array}{c}\text { Variável } \\
\text { Leste/Oeste } \\
\text { Fixa_NORTE }\end{array}$ & 2 & 36 & $\begin{array}{c}\text { 15,68MW } \\
\text { /Ano }\end{array}$ \\
\hline
\end{tabular}

Tabela 1

As inclinações usadas no sistema tracker obedeceram as sugestões do PVsyst, ou seja, $10^{\circ}$ Norte com rotação possível de $-60^{\circ}$ a $+60^{\circ}$ (Leste/Oeste) e azimute $0^{\circ}$.

A determinação da trajetória solar pode ser obtida por meio de simulação, utilizando-se programas específicos, nos quais se encontram programadas as equações que determinam sua trajetória e que plotam a carta solar para uma dada latitude e longitude dentro do globo terrestre. Essa trajetória pode ser obtida também diretamente a partir de cálculos baseados nas equações que determinam a posição do sol em qualquer latitude, dia e hora do ano (OLIVEIRA, 2013) [5].

Para nosso estudo, o software arduino será desenvolvido para fazer movimentações a cada hora com passos de $15 \% / \mathrm{h}$ no período de; $6 \mathrm{~h}$ da manhã até às $18: 00 \mathrm{~h}$. Ao termino do movimento o sistema voltará ao seu estágio inicial, pois terá um contra-peso para isto e batedores para sustentação em repouso. No conjunto do tracker não usaremos engrenagens e sim um sistema com tubulação vazada livre para o movimento e sistemas com correias do tipo cinto de segurança, que por sua vez concentrarão o esforço após cada movimento portanto, deixando o motor de passo desligado, vindo a ligar após $1 \mathrm{~h}$ para o próximo movimento. Como o processo é feito com contrapeso e sistema de batedores, caso haja algum problema com o processo (Queima do motor ou danificação do arduino) o proprietário basta soltar a correia que o sistema ficará na sua posição ótima até que o problema seja resolvido.

\section{RESULTADOS E DISCUSSÃO}

Faremos uma análise comparativa dos dois processos e veremos o quanto de ganho teremos com o sistema tracker sem engrenagens.

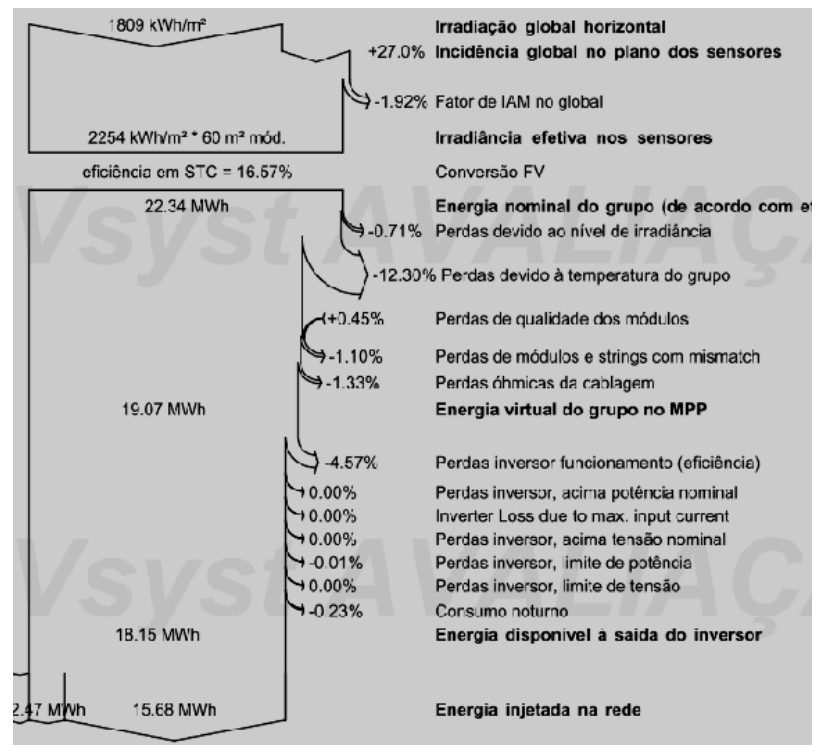

Figura 1: Diagrama de Perdas do Sistema COM tracker durante o ano.

Fonte: Autor (2019). 


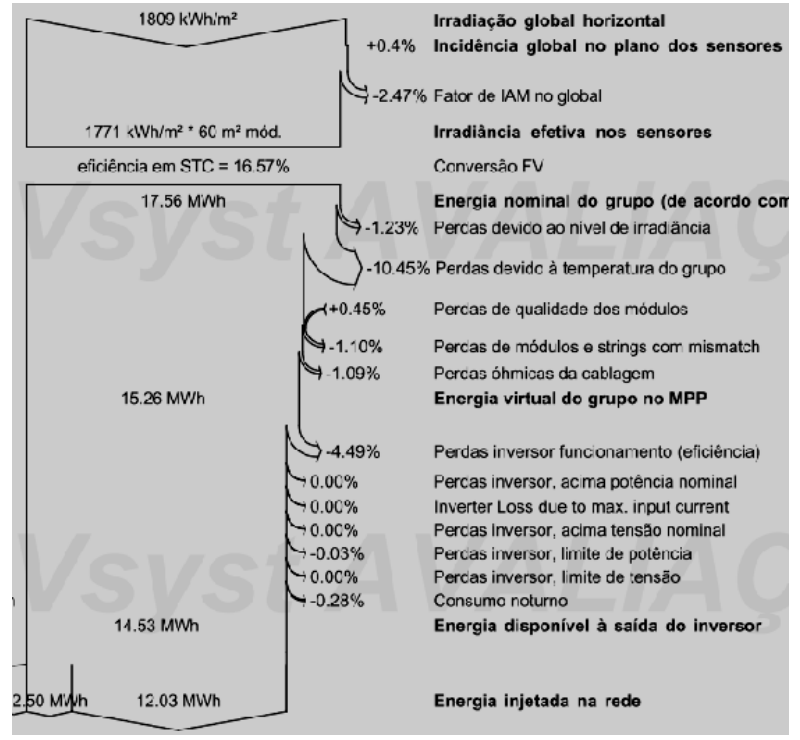

Figura 2: Diagrama de Perdas do Sistema SEM tracker durante o ano.

Fonte: Autor (2019).

$\mathrm{Na}$ Figura 1 observamos o diagrama de perdas onde temos uma projeção de um ano do sistema fotovoltaico com o sistema tracker, destacando-se como resultado final o valor de $15,68 \mathrm{MWh}$ que é a energia injetada na rede depois de todas as perdas, onde podemos também obter e destacar os percentuais das perdas de energia fotovoltaica devido a irradiância que foi de $-1.92 \%$, as perdas de energia fotovoltaica decorrente a temperatura que foi de $-12,30 \%$.

Já na Figura 2 , observamos os mesmos dados no diagrama de perdas para o mesmo período onde obtivemos os seguintes parâmetros: $12,03 \mathrm{MWh}$ de energia ejetada na rede, $-2,47 \%$ de perdas devido à irradiância e $-10,45 \%$ devido à perdas por temperatura.

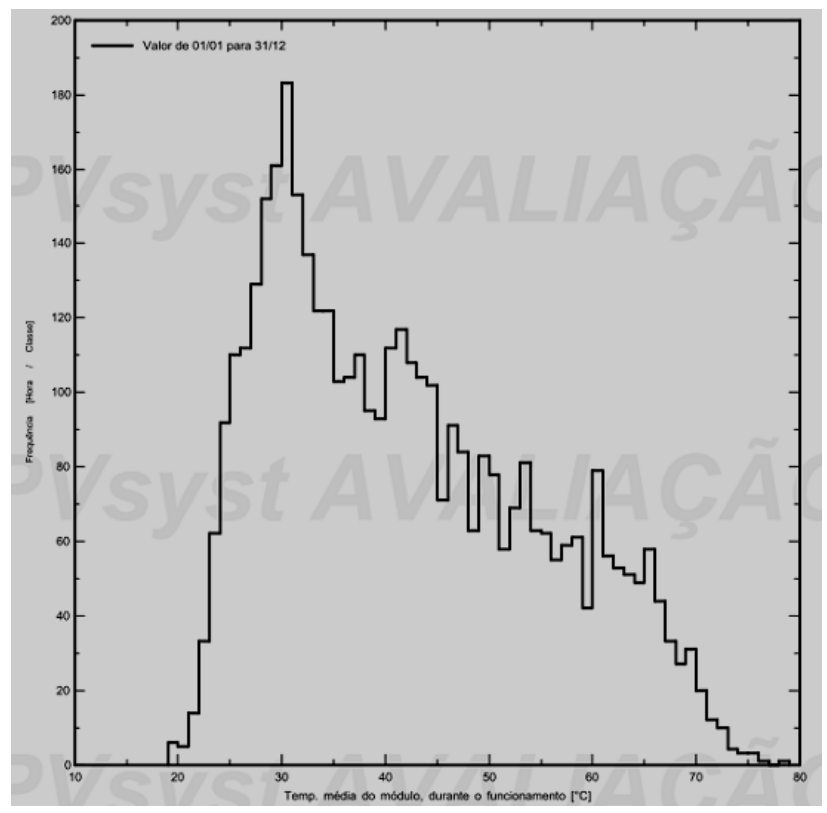

Figura 3: Diagrama de Distribuição da temperatura do Grupo para o Sistema COM tracker durante o ano.

Fonte: Autor (2019).

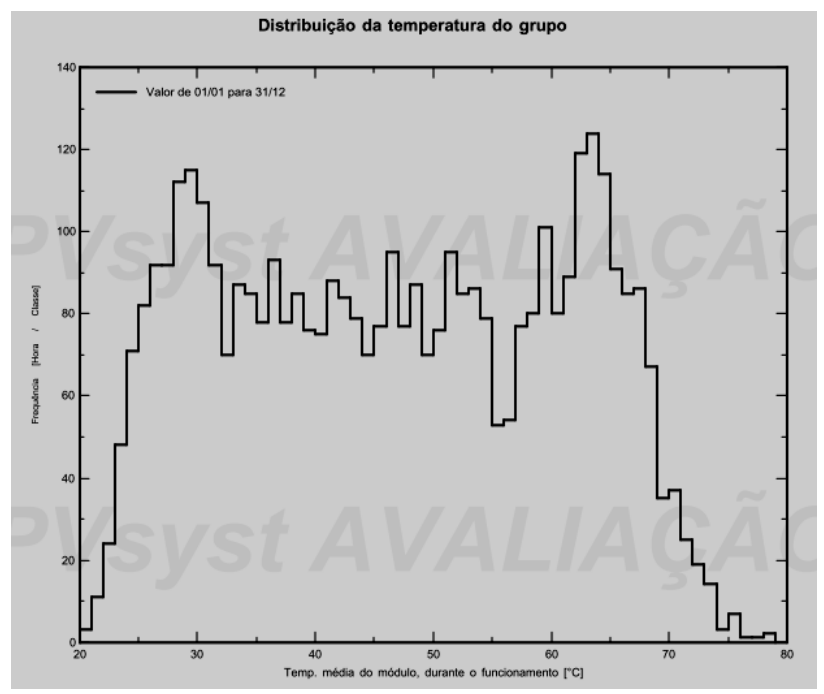

Figura 4: Diagrama de Distribuição da temperatura do Grupo para o Sistema SEM tracker durante o ano.

Fonte: Autor (2019).

Nas figuras 3 e 4 , vemos nitidamente um aumento na freqüência de temperaturas mais altas com o sistema Tracker, espera-se que essas temperaturas sejam mais reduzidas, uma vez que, 0 ambiente onde as placas seriam instaladas é bastante ventilado. 


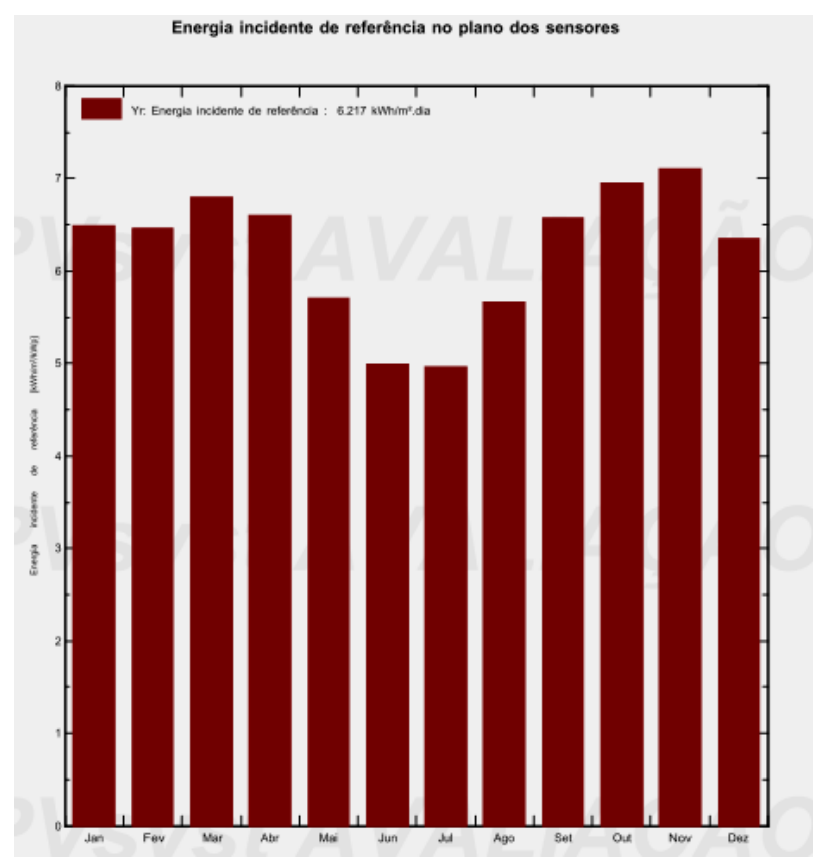

Figura 5: Diagrama de Energia incidente de referência

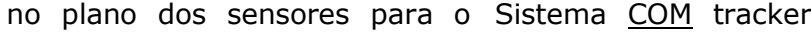
durante o ano.

Fonte: Autor (2019).

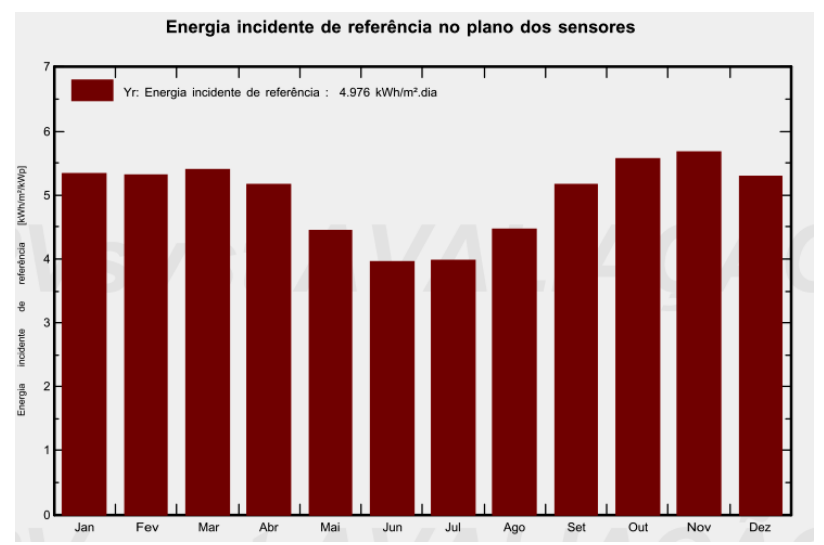

Figura 6: Diagrama de Energia incidente de referência no plano dos sensores para o Sistema SEM tracker durante $o$ ano.

Fonte: Autor (2019).

Podemos ver nestes gráficos das figuras 5 e 6 um nível de geração maior de energia em TODOS os meses para o sistema com Tracker.

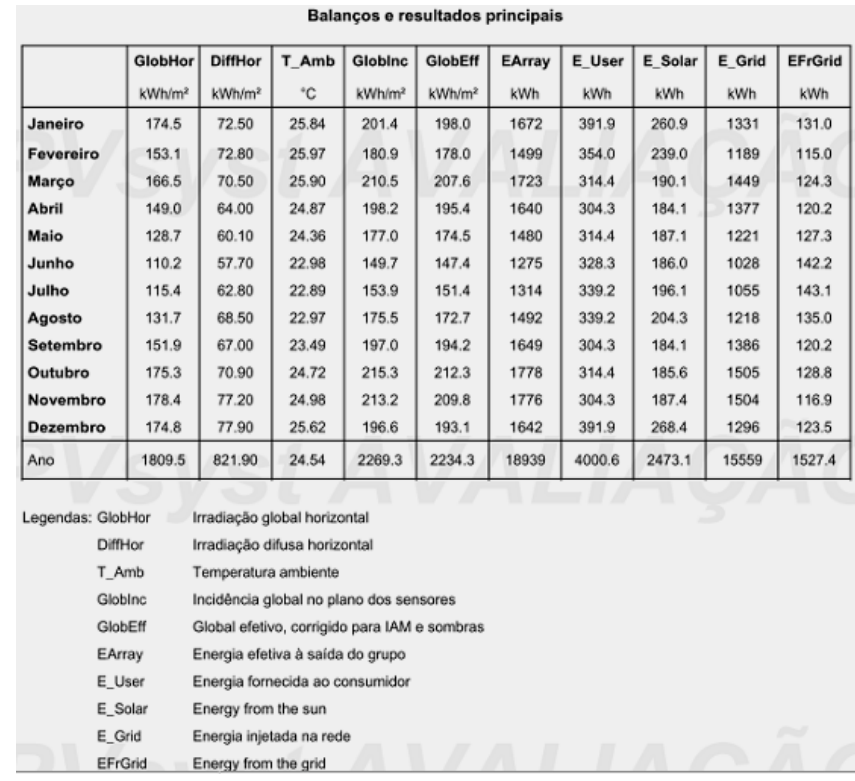

Figura 7: Tabela de Balanços e resultados principais para o Sistema COM tracker durante o ano.

Fonte: Autor (2019).

\begin{tabular}{|c|c|c|c|c|c|c|c|c|c|c|}
\hline \multicolumn{11}{|c|}{ Balanços e resultados principais } \\
\hline & $\begin{array}{l}\text { GlobHor } \\
\mathrm{kWh} / \mathrm{m}^{2}\end{array}$ & $\begin{array}{l}\text { DiffHHor } \\
\mathrm{kWh} / \mathrm{m}^{2}\end{array}$ & \begin{tabular}{|c|} 
T_Amb \\
${ }^{\circ} \mathrm{C}$
\end{tabular} & \begin{tabular}{l|} 
Globinc \\
$\mathrm{kWh} / \mathrm{m}^{2}$
\end{tabular} & $\begin{array}{l}\text { GlobEff } \\
\mathrm{kWh} / \mathrm{m}^{2}\end{array}$ & $\begin{array}{c}\text { EArray } \\
k W h\end{array}$ & $\begin{array}{c}\text { E_User } \\
\mathrm{kWh}\end{array}$ & $\begin{array}{c}\text { E_Solar } \\
\mathrm{kWh}\end{array}$ & $\begin{array}{c}\text { E_Grid } \\
\mathrm{kWh}\end{array}$ & $\begin{array}{c}\text { EFrGrid } \\
\mathrm{kWh}\end{array}$ \\
\hline Janeiro & 174.5 & 72.50 & 25.84 & 165.0 & 160.8 & 1376 & 391.9 & 264.3 & 1045 & 127.6 \\
\hline Fevereiro & 153.1 & 72.80 & 25.97 & 149.0 & 145.3 & 1242 & 354.0 & 241.2 & 942 & 112.8 \\
\hline Março & 166.5 & 70.50 & 25.90 & 167.2 & 163.3 & 1387 & 314.4 & 191.6 & 1129 & 122.8 \\
\hline Abril & 149.0 & 64.00 & 24.87 & 154.8 & 151.2 & 1297 & 304.3 & 185.8 & 1050 & 118.5 \\
\hline Maio & 128.7 & 60.10 & 24.36 & 137.8 & 134.4 & 1165 & 314.4 & 189.1 & 921 & 125.3 \\
\hline Junho & 110.2 & 57.70 & 22.98 & 118.9 & 115.9 & 1019 & 328.3 & 189.2 & 780 & 139.1 \\
\hline Julho & 115.4 & 62.80 & 22.89 & 123.5 & 120.4 & 1060 & 339.2 & 199.1 & 810 & 140.1 \\
\hline Agosto & 131.7 & 68.50 & 22.97 & 138.4 & 134.9 & 1184 & 339.2 & 205.8 & 923 & 133.5 \\
\hline Setembro & 151.9 & 67.00 & 23.49 & 155.1 & 151.5 & 1312 & 304.3 & 185.5 & 1064 & 118.8 \\
\hline Outubro & 175.3 & 70.90 & 24.72 & 172.3 & 168.2 & 1431 & 314.4 & 190.2 & 1171 & 124.2 \\
\hline Novembro & 178.4 & 77.20 & 24.98 & 169.9 & 165.2 & 1413 & 304.3 & 189.2 & 1157 & 115.1 \\
\hline Dezembro & 174.8 & 77.90 & 25.62 & 164.3 & 159.9 & 1374 & 391.9 & 272.4 & 1036 & 119.5 \\
\hline Ano & 1809.5 & 821.90 & 24.54 & 1816.1 & 1771.2 & 15261 & 4000.6 & 2503.3 & 12028 & 1497.3 \\
\hline \multicolumn{11}{|c|}{ Legendas: GlobHor Irradiaçao global horizontal } \\
\hline \multicolumn{2}{|c|}{ DiffHor } & \multicolumn{9}{|c|}{ Irradiaçắo difusa horizontal } \\
\hline \multicolumn{2}{|c|}{ T_Amb } & \multicolumn{9}{|c|}{ Temperatura ambiente } \\
\hline \multicolumn{2}{|c|}{ Globlnc } & \multicolumn{9}{|c|}{ Incidéncia global no plano dos sensores } \\
\hline \multicolumn{2}{|c|}{ GlobEH } & \multicolumn{9}{|c|}{ Global efetivo, corrigido para IAM e sombras } \\
\hline \multicolumn{2}{|c|}{ EArray } & \multicolumn{9}{|c|}{ Energia efetiva à saida do grupo } \\
\hline \multicolumn{2}{|c|}{ E_User } & \multicolumn{9}{|c|}{ Energia fornecida ao consumidor } \\
\hline \multicolumn{2}{|c|}{ E_Solar } & \multicolumn{9}{|c|}{ Energy from the sun } \\
\hline \multicolumn{2}{|c|}{ E_Grid } & \multicolumn{9}{|c|}{ Energia injetada na rede } \\
\hline & & Energy from & the grid & & & & & & & \\
\hline
\end{tabular}

Figura 8: Tabela de Balanços e resultados principais para o Sistema SEM tracker durante o ano.

Fonte: Autor (2019).

Fazendo uma comparação dos dados das figuras 7 e 8 , vemos claramente as vantagens de se usar o sistema tracker para 0 projeto em questão. 


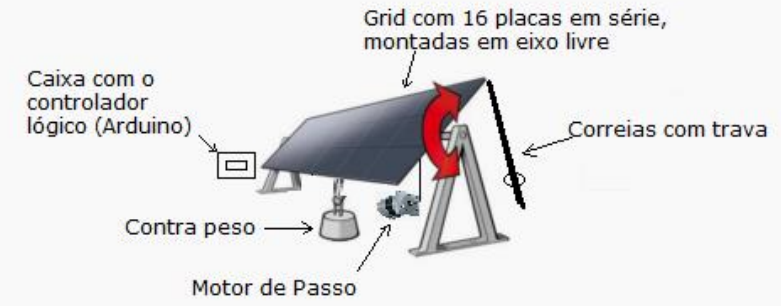

Figura 9: Esquema detalhado do sistema tracker a ser usado no projeto.

Fonte: Desenvolvimento de um Sistema para Rastreamento Solar- (Tese de Mestrado)_Com adaptações.

\section{CONCLUSÕES}

Demonstrada a análise comparativa de geração com sistema fixo e com a aplicação do Tracker sem engrenagens, pudemos perceber um aumento de exatos $29,4 \%$ de energia entregue ao consumidor/concessionária. Esse ganho aproximado de $30 \%$ na geração, pode ter uma pequena redução devido a elevação indesejada da temperatura das placas em virtude da maior incidência dos raios solares nas mesmas. Sendo assim, o mais recomendado é que o local e a forma de instalação proporcione uma boa ventilação para que possam dissipar calor facilmente e evitar perdas por excesso de temperatura. A fim de impedir problemas e manter a eficiência do processo, é necessária a manutenção regular do sistema, sendo uma boa prática a elaboração de plano de manutenção que atenda além das especificações do fabricante, as especificações do projeto. Este sistema inovador mostra que podemos aumentar nosso aproveitamento da planta solar sem muitos gastos e com pouquíssima manutenção tanto corretiva quanto preventiva, uma vez que, o sistema funciona com poucas partes móveis que não exigem reparos ou processo de manutenção relevante. Ressaltando ainda que; caso haja qualquer problema no processo, basta que o proprietário solte as correias que estão acopladas ao sistema de travamento e ao motor para que o sistema fique gerando em sua configuração ótima quando na posição fixa.

\section{Referências}

[1] Matriz Energética e Elétrica. Empresa de Pesquisa Energética (EPE). Disponível em: $<$ http://epe.gov.br/pt/abcdenergia/matrizenergetica-e-eletrica> Acesso em:15 de Maio 2019.

[2] Agência Nacional de Energia Elétrica. Resolução Normativa No 482, de 17 de Abril de 2012. Disponível em:

<http://www2.aneel.gov.br/cedoc/ren20124 82.pdf> Acesso em:18 de Maio 2019.

[3] Blog Filipeflop. O que é Arduino. Disponível em:

$<$ https://www.filipeflop.com/blog/o-que-earduino/> Acesso em: 18 de Maio de 2019.

[4] Kalatec Automação. O que é o Motor de Passo. Disponível em:

<https://www.kalatec.com.br/o-que-e-ummotor-de-passo/> Acesso em 18 de Maio de 2019.

[5] OLIVEIRA, L. B.; NASCIMENTO NETO, J. A. Desenvolvimento de Medidor de Trajetória Solar e Aplicado na Cidade de Cajazeiras. In: REUNIÃO ANUAL DA SOCIEDADE BRASILEIRA PARA O PROGRESSO DA CIÊNCIA, 65., 2013, Recife. Anais... São Paulo: SBPC/UFPE, 2013. 